\title{
Test-meal palatability is associated with overconsumption but better represents preceding changes in appetite in non-obese males
}

\author{
Kevin Deighton $^{1 *}$, James Frampton ${ }^{2}$ and Javier T. Gonzalez ${ }^{2}$ \\ ${ }^{1}$ Institute for Sport, Physical Activity \& Leisure, Leeds Beckett University, Leeds LS6 3QS, UK \\ ${ }^{2}$ Department for Health, University of Bath, Bath BA2 7AY, UK \\ (Submitted 7 March 2016 - Final revision received 13 May 2016 - Accepted 23 June 2016 - First published online 1 August 2016)
}

\section{Abstract}

Single-course, ad libitum meals are recommended for the assessment of energy intake within appetite research. This study represents the first investigation of the comparative sensitivity of two single-course, ad libitum meals designed to differ in palatability. We conducted two experiments using a preload study design. All protocols were identical except for the energy content of the preloads (Expt 1: 579 and $1776 \mathrm{~kJ}$; Expt 2: 828 and $4188 \mathrm{~kJ}$ ). During each experiment, ten healthy men completed four experimental trials constituting a low- or high-energy preload beverage, a 60-min intermeal interval and consumption of a pasta-based or a porridge-based, ad libitum meal. Appetite ratings were measured throughout each trial, and palatability was assessed after food consumption. Preload manipulation did not influence appetite $(P=0.791)$ or energy intake $(P=0.561)$ in Expt 1 . Palatability and energy intake were higher for the pasta meal than for the porridge meal in both experiments (palatability $P \leq 0.002$; energy intake $P \leq 0.001$ ). In Expt 2 , consumption of the high-energy preload decreased appetite $(P=0.051)$ and energy intake $(P=0.002)$. Energy compensation was not significantly different between pasta and porridge meals $(P=0 \cdot 172)$, but was more strongly correlated with preceding changes in appetite at the pasta meal $(r-0 \cdot 758 ; P=0 \cdot 011)$ than the porridge meal $(r-0 \cdot 498$; $P=0.143)$. The provision of a highly palatable, pasta-based meal produced energy intakes that were more representative of preceding appetite ratings, but the moderately palatable, porridge-based meal produced more ecologically valid energy intakes. Ad libitum meal selection and design may require a compromise between sensitivity and ecological validity.

\section{Key words: Energy intake: Preloads: Energy compensation: Sensitivity}

The increase in obesity prevalence during recent decades has stimulated an abundance of research into the regulation of appetite and energy balance in humans. This research frequently includes the objective measurement of energy intake during ad libitum meals in response to nutritional ${ }^{(1,2)}$, pharmaceutical $^{(3,4)}$ and exercise interventions $^{(5,6)}$. Such monitoring of energy intakes under laboratory conditions is recommended because of the dubious accuracy of self-reported measures ${ }^{(7,8)}$, and a range of ad libitum meals have demonstrated high levels of repeatability in quantifying energy intakes $^{(9-13)}$. However, despite the prevalent use of ad libitum feeding, there has been little investigation into the sensitivity of these meals to reflect changes in appetite, and only one study to date has compared the sensitivity of commonly used ad libitum meals. In this regard, Wiessing et al. ${ }^{(14)}$ recently demonstrated a similar energy compensation of approximately $28 \%$ in response to a high-energy $v$. low-energy preload when assessing energy intake via an ad libitum buffet meal and single-course, pasta-based meal. However, both meals promoted overconsumption with mean intakes $>4500 \mathrm{~kJ}$ at each meal after the low-energy preload.

Single-course meals are recommended for the assessment of ad libitum energy intake because of concerns that buffet meals delay satiation and promote overconsumption, thereby not reflecting the habitual intakes of participants ${ }^{(7)}$. However, overconsumption during single-course, pasta-based meals is commonly reported in the literature, with mean intakes ranging from approximately 3200 to $6400 \mathrm{~kJ}$ in a range of participant populations $^{(1,14-20)}$. Such large intakes are likely to be due to the high palatability of pasta-based ad libitum meals ${ }^{(14,21)}$. It has previously been demonstrated that increasing the palatability of $a d$ libitum meals can enhance appetite during feeding, induce overconsumption and reduce the sensitivity of the meal to detect previous changes in appetite ${ }^{(22)}$. Subsequently, it seems plausible that overconsumption during pasta-based meals may contribute to the dissociations observed between appetite ratings and food intake responses in previous studies ${ }^{(1,15,18)}$.

Recent studies by Corney et al. ${ }^{(23,24)}$ have used an ad libitum porridge meal to assess energy intake and have reported mean intakes of approximately $2500 \mathrm{~kJ}$ after an overnight fast in healthy, young men. These intakes are substantially lower than those reported from pasta meals within similar populations ${ }^{(15-18)}$, are more representative of expected habitual intakes (increasing external validity) and may produce greater sensitivity to previous changes in appetite by reducing overconsumption (enhancing precision). However, because of large individual differences in

Abbreviations: HE, high energy; LE, low energy.

* Corresponding author: Dr K. Deighton, email K.Deighton@leedsbeckett.ac.uk 
energy intake during ad libitum feeding combined with the subjectivity of appetite perceptions, direct comparisons within subjects are essential for appropriate assessment of appetite and energy intake responses to an intervention ${ }^{(7)}$.

Thus, the purpose of this study was to compare the sensitivity of a pasta-based $v$. a porridge-based ad libitum meal for the assessment of energy intake. This represents the first comparison of two commonly used single-course, ad libitum meals and provides guidance on the selection of ad libitum meals for future research studies. We hypothesised that ad libitum energy intake at the porridge-based meal would be more ecologically valid and more representative of preceding appetite ratings than energy intake at the pasta-based meal.

\section{Methods \\ Study design}

This investigation included two experiments, which were conducted according to the guidelines laid down in the Declaration of Helsinki. Both experiments involved a preload study design to investigate the influence of ad libitum meal composition on the compensatory energy intake response to different energy preloads. The experimental protocols were identical, except for the energy content of the preloads. Expt 1 was conducted at the University of Bath and it compared the effects of a 579 and $1776 \mathrm{~kJ}$ preload. Expt 2 was conducted at Leeds Beckett University and it compared the effects of an 828 and $4188 \mathrm{~kJ}$ preload. The use of different preloads in each experiment enabled comparisons to be made regarding the effects of moderate and large differences in preload energy content. Each experiment was approved by the Institutional Ethics Advisory Committee for the university at which the experimental testing was performed, and written informed consent was obtained from all participants.

\section{Participants and standardisation}

Study participants were non-smokers, not taking any medication, weight stable for at least 6 months before participation and were not dieting. Participants had no known history of CVD/ metabolic disease, were classified as unrestrained eaters ${ }^{(25)}$ and were recreationally active.

In both experiments, participants completed a food diary detailing all foods and drinks consumed in the $24 \mathrm{~h}$ before their first experimental trial and repeated this before each subsequent trial. Alcohol, caffeine and strenuous physical activity were not permitted during this period. All trials commenced between 08.00 and 09.00 hours after an overnight fast of at least $10 \mathrm{~h}$, and participants exerted themselves minimally when travelling to the laboratory, using motorised transport when possible. Verbal confirmation of dietary and exercise standardisation was obtained at the beginning of each experimental trial.

\section{Experimental protocol}

For each experiment, ten healthy men participated in four experimental trials separated by a minimum of $72 \mathrm{~h}$ in a randomised, semi-double blind (blinded to the preload composition but not the test meal), cross-over design. The four trials constituted a low-energy (LE) or high-energy (HE) preload, followed by an ad libitum test meal that was either pasta based or porridge based. Anthropometric measurements, screening for eating behaviours ${ }^{(25)}$, habitual physical activity levels and verbal confirmation of the acceptability of the foods to be provided during the study were obtained immediately before the first experimental trial. Habitual consumption of pasta-based and porridge-based meals was assessed using an eight-point scale ranging from 'almost never' to ' $>2$ meals/d'.

Upon arrival to the laboratory for each experimental trial, participants completed a baseline appetite visual analogue scale before consuming a low- or high-energy preload beverage. Participants were instructed to consume the beverage within $5 \mathrm{~min}$, and a 60-min intermeal interval commenced upon the first mouthful of the beverage in accordance with Almiron-Roig et $a l .{ }^{(26)}$. Participants rested within the laboratory (sitting, reading or listening to music) throughout the intermeal interval, and were provided with an ad libitum pasta-based or porridgebased meal at $60 \mathrm{~min}$.

\section{Preloads}

The preload beverages were matched for macronutrient composition and were designed to closely align with the UK dietary guidelines for macronutrient proportions (58\% carbohydrate, $26 \%$ fat, $16 \%$ protein). The preloads consisted of water, single cream (Tesco), maltodextrin (MyProtein), whey protein isolate (MyProtein) and vanilla flavouring (MyProtein). These beverages were comparable with those used in previous studies ${ }^{(19)}$. The energy content of the preload beverages was 579 and $1776 \mathrm{~kJ}$ in Expt 1 and 828 and $4188 \mathrm{~kJ}$ in Expt 2. All preload beverages weighed $550 \mathrm{~g}$ and were distributed evenly between two 568-ml glasses in order to disguise any subtle differences in volume. All beverages were consumed by participants in isolation. The preloads were prepared by a third party external to the study, and both the researcher and participant were asked to identify which beverage they thought had been consumed at the end of each trial. All participants were fully unblinded upon completion of the experiment.

\section{Appetite and palatability assessment}

Appetite perceptions (hunger, satisfaction, fullness and prospective food consumption (PFC)) were assessed at baseline and every $15 \mathrm{~min}$ during both experiments using 100-mm visual analogue scales with descriptors anchored at each end describing the extremes (e.g. 'I am not hungry at all'/'I have never been more hungry') ${ }^{(27)}$. Participants rated their appetite perceptions by placing a mark across each line on paper, and participants were not able to refer to their previous ratings when completing the appetite scales. The scales were analysed by measuring the horizontal distance from the left-hand side of the continuum to the point on the line indicated by the participant. Each visual analogue scale was measured twice to ensure accuracy. A composite appetite score was calculated for each time point as the mean value of the four appetite 
perceptions after inverting the values for satisfaction and fullness ${ }^{(28)}$. Palatability ratings (visual appeal, smell, taste, aftertaste and pleasantness) were obtained for the preloads and ad libitum meals immediately after consumption ${ }^{(27)}$. A composite palatability score was calculated as the mean value of the palatability subscales.

\section{Ad libitum meals}

The ad libitum meals were matched for macronutrient content and were designed to closely align with the UK dietary guidelines for macronutrient proportions ( $52 \%$ carbohydrate, $34 \%$ fat and $14 \%$ protein). The meals were also matched for energy density $(8.45 \mathrm{~kJ} / \mathrm{g})$. The pasta-based meal consisted of penne pasta (Tesco), Cheddar cheese (Tesco), tomato sauce (Tesco) and olive oil (Tesco) in accordance with previous research $^{(15,16)}$. Pasta was cooked for $15 \mathrm{~min}$ in unsalted water at $700 \mathrm{~W}$ before being mixed with the remaining ingredients and re-heated for $2 \mathrm{~min}$ at $700 \mathrm{~W}$. The porridge-based meal consisted of rolled oats (Tesco), whole milk (Tesco), double cream (Tesco), maltodextrin (MyProtein) and whey protein isolate (MyProtein). The oats were cooked in the microwave with milk and double cream for $2 \mathrm{~min}$ at $700 \mathrm{~W}$ before being mixed with the remaining ingredients.

Participants consumed the ad libitum meals in isolation in order to prevent any social influence affecting food intake. Participants were provided with a bowl of the respective meal, and this was replaced by an investigator before the participant had emptied it and with minimal interaction. Each portion of the porridge-based meal weighed $300 \mathrm{~g}$, and each portion of the pasta-based meal weighed $430 \mathrm{~g}$ before consumption; three bowls of the respective meal were prepared for each trial in accordance with previous research ${ }^{(15)}$, which met the requirements of all participants during the trials. No time limit was set for eating, and participants were instructed to eat until 'comfortably full'. Subsequently, participants determined the point of meal termination and were asked to leave the feeding area and to inform the researcher once they felt 'comfortably full'. Food intake was determined as the weighted difference in food before and after eating. Water was available ad libitum during the participants' first trial and standardised for each subsequent trial. Energy compensation was calculated using the following equation:

Energy compensation (\%)

$=\left(\left(\right.\right.$ energy intake $_{\text {low energy preload }}-$ energy intake $\left.{ }_{\text {high energy preload }}\right)$

/energy difference between preloads) $\times 100$.

\section{Statistical analysis}

Data for each experiment were analysed separately using IBM SPSS statistics version 19 for Windows. Total AUC values were calculated for appetite perceptions using the trapezoidal method. Repeated measures, two-way ANOVA (preload $\times$ meal) was used to assess differences in energy intake, composite palatability scores and AUC values for composite appetite scores between the trials. Pearson's product-moment correlation coefficient was used to examine the relationship between energy intake and preceding appetite ratings. This included correlations between the change in appetite scores and the percentage energy compensation in response to the highenergy preload compared with the low-energy preload in order to determine the utility of the test meals to reflect changes in appetite. Wilcoxon's signed-rank was used to assess differences between the habitual consumption of pasta-based and porridge-based meals. Statistical significance for this study was accepted at $P \leq 0.05$. Participant characteristics and data in the text are presented as mean values and standard deviations. All other results are presented as mean values and $95 \% \mathrm{CI}$. A sample size of ten participants was determined to be sufficient to detect an energy compensation of $40 \%$ in Expt 1 and $15 \%$ in Expt 2, based on previous data from Corney et $a l .{ }^{(23)}$. This calculation was performed using $\mathrm{G}^{*}$ power with an $\alpha$ value of $5 \%$ and a power of $80 \%{ }^{(29)}$. Individual compensatory responses are plotted within the figures to allow further examination of the findings, and the results of each experiment are presented separately to ensure clarity.

\section{Results}

Expt 1

Participant characteristics. Participant characteristics were as follows: age 22 (SD 1) years, height 1.80 (SD 0.06) $\mathrm{m}$, body mass $81 \cdot 1$ (sD 7.9) $\mathrm{kg}$ and BMI $24.8(\mathrm{sD} 1.6) \mathrm{kg} / \mathrm{m}^{2}$. There was no significant difference in the habitual consumption of pasta-based and porridge-based meals $(P=0.917)$ with the same median intake of 1 meal/week. Habitual consumption of pasta-based meals ranged from 'almost never consumed' to '5-6 meals/week', whereas habitual consumption of porridgebased meals ranged from 'almost never consumed' to ' 1 meal/d'.

Energy intake. Two-way ANOVA revealed higher ad libitum energy intake during the pasta meal compared with the porridge meal $(P<0.0005)$ but no difference between the 579 and $1776 \mathrm{~kJ}$ preloads $(P=0.561)$ (Fig. 1(a)). There was no significant difference in energy compensation between test meals $(P=0.922)$ (Fig. 1(b)).

Appetite and palatability ratings. Two-way ANOVA demonstrated similar results for each appetite perception with no significant differences between preloads and test meals for hunger (preload: $P=0.694$; meal: $P=0.928$ ), satisfaction (preload: $P=0.420$; meal: $P=0.239$ ), fullness (preload: $P=0.338$; meal: $P=0.233$ ) or PFC (preload: $P=0.241$; meal: $P=0.862$ ). Subsequently, composite appetite scores are presented for clarity.

Composite appetite scores did not differ between trials at baseline $(P=0 \cdot 421)$. Two-way ANOVA revealed no significant difference in composite appetite AUC between the 579 and the $1776 \mathrm{~kJ}$ preload trials $(P=0.791)$. Similarly, there was no difference in appetite scores between the pasta and the porridge trials $(P=0.523$; LE porridge 70 (SD 10), LE pasta 64 (SD 9), HE porridge 65 (SD 14), HE pasta 68 (sD 14)) (Fig. 2). 

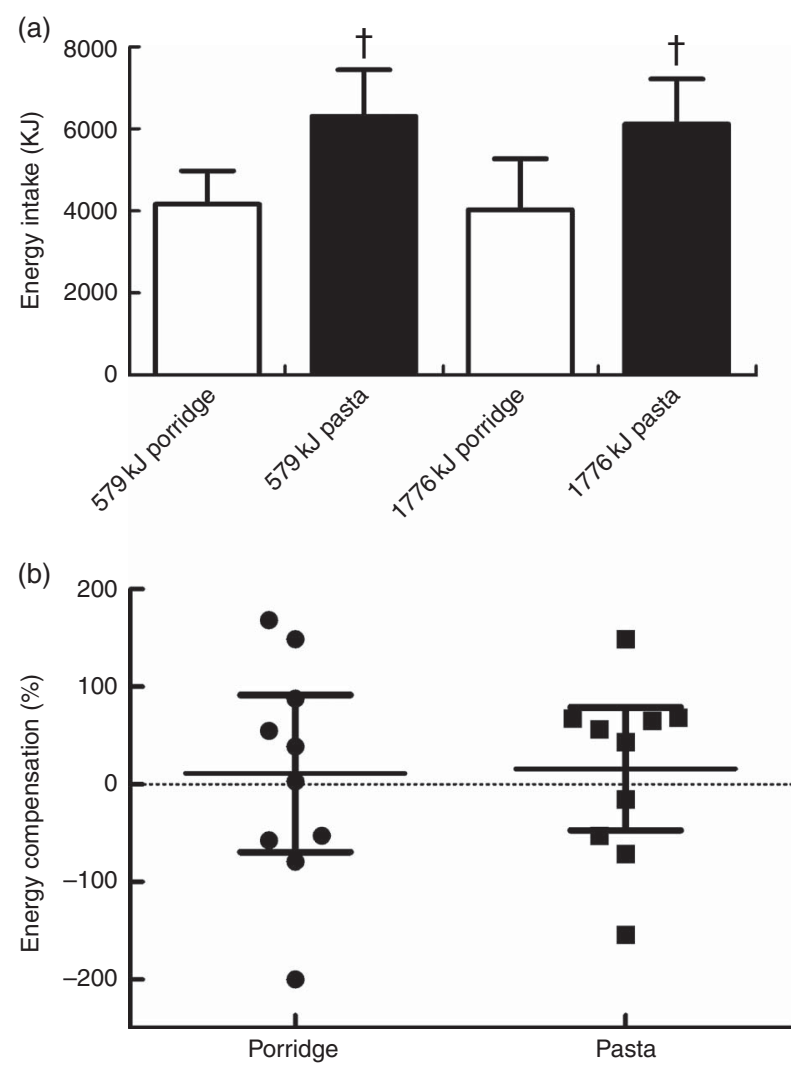

Fig. 1. Energy intake (a) and energy compensation (b) for Expt 1. $\dagger$ Significantly different between test meals. Values are means $(n 10)$ and $95 \% \mathrm{Cl}$.

Two-way ANOVA demonstrated no significant differences in composite palatability scores for the high-energy preload compared with the low-energy preload $(P=0.136)$. The palatability response to preloads was not different during the pasta and porridge trials $(P=0 \cdot 218)$. Composite palatability scores for the test meals were significantly higher for the pasta meal compared with the porridge meal $(P=0 \cdot 001)$. The palatability response to the test meals was not different during the high- and low-energy preload trials $(P=0.431)$ (Fig. 3).

The preload beverage was correctly identified by participants in twenty-one of the forty trials and by the researcher in five of the forty trials.

Correlations. Composite appetite AUC values were not significantly correlated with energy intake in any of the four trials (all $r<0.438 ; P>0.205$ ). Energy compensation at the ad libitum meals was not significantly correlated with the change in AUC or 60-min composite appetite scores between the 579 and the $1776 \mathrm{~kJ}$ preloads (pasta AUC: $r 0.077, P=0.832$; pasta $60 \mathrm{~min}$ : $r-0.497, P=0.143$; porridge AUC: $r-0.452$, $P=0 \cdot 190$; porridge $60 \mathrm{~min}: r-0 \cdot 385, P=0 \cdot 272$ ) (Fig. 2).

Expt 2

Participant characteristics. Participant characteristics were as follows: age 21 (sD 4) years, height $1.80(\mathrm{sD} 0.05) \mathrm{m}$, body mass
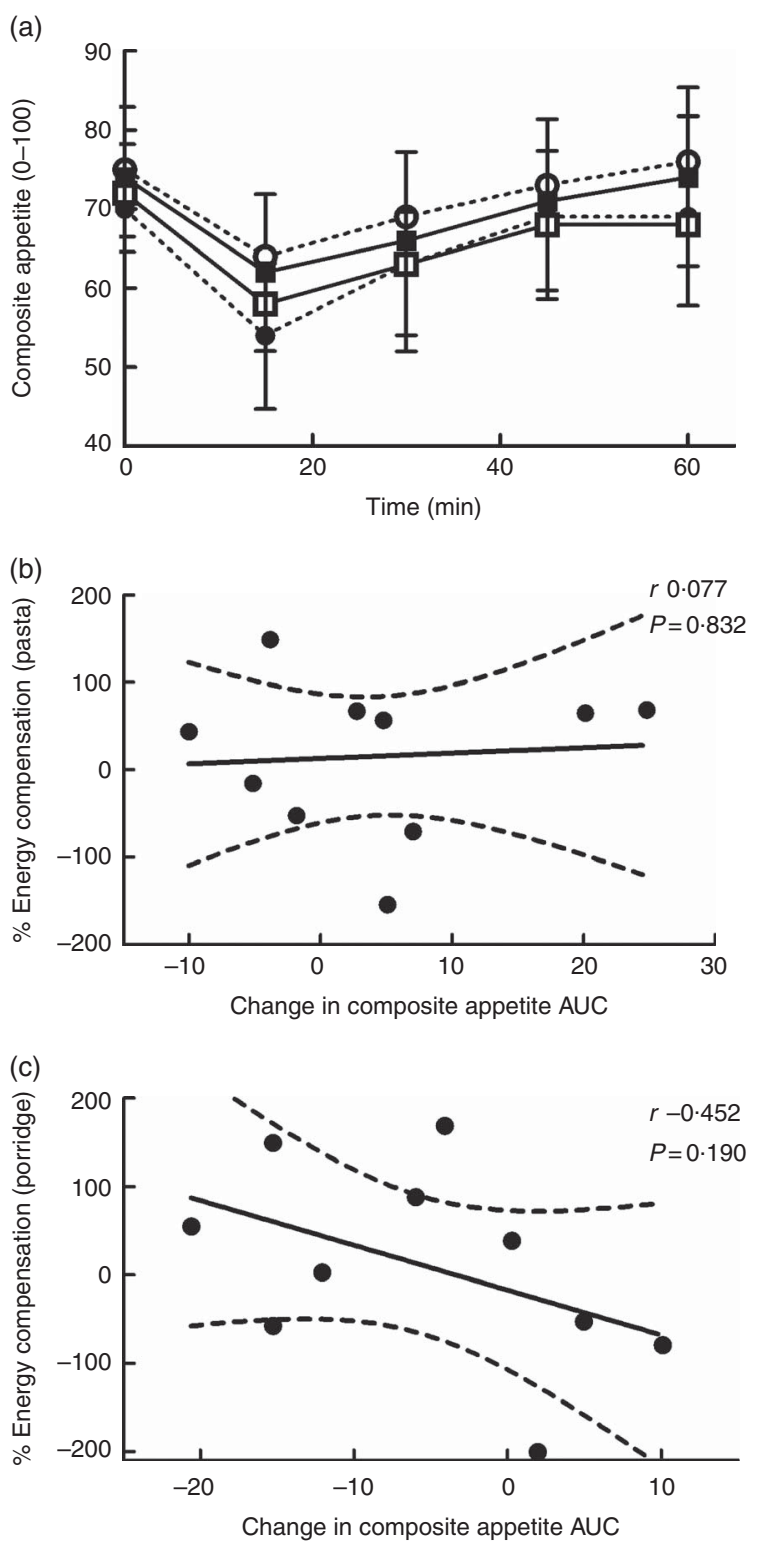

Fig. 2. Composite appetite scores (a) in the $579 \mathrm{~kJ}$ porridge (O), $579 \mathrm{~kJ}$

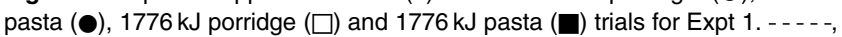
The low-energy preload trials. Values are means $(n 10)$ and $95 \% \mathrm{Cl}$. Linear correlation with $95 \% \mathrm{Cl}$ between the change in composite appetite AUC after the 1776 v. $579 \mathrm{~kJ}$ preload and energy compensation for the pasta meal (b) and porridge meal (c).

77.2 (sD 6.4) $\mathrm{kg}$ and BMI 24.2 (SD 2.3) kg/m². Habitual consumption of pasta-based meals was significantly higher than porridge-based meals $(P=0.014)$ with median intakes of '2-4 meals/week' and '1 meal/week', respectively. Habitual consumption of pasta-based meals ranged from ' 1 meal/week' to '2-4 meals/week', whereas habitual consumption of porridge-based meals ranged from 'almost never consumed' to '2-4 meals/week'.

Energy intake. Two-way ANOVA demonstrated higher ad libitum energy intake after the $828 \mathrm{~kJ}$ preload compared 
(a)
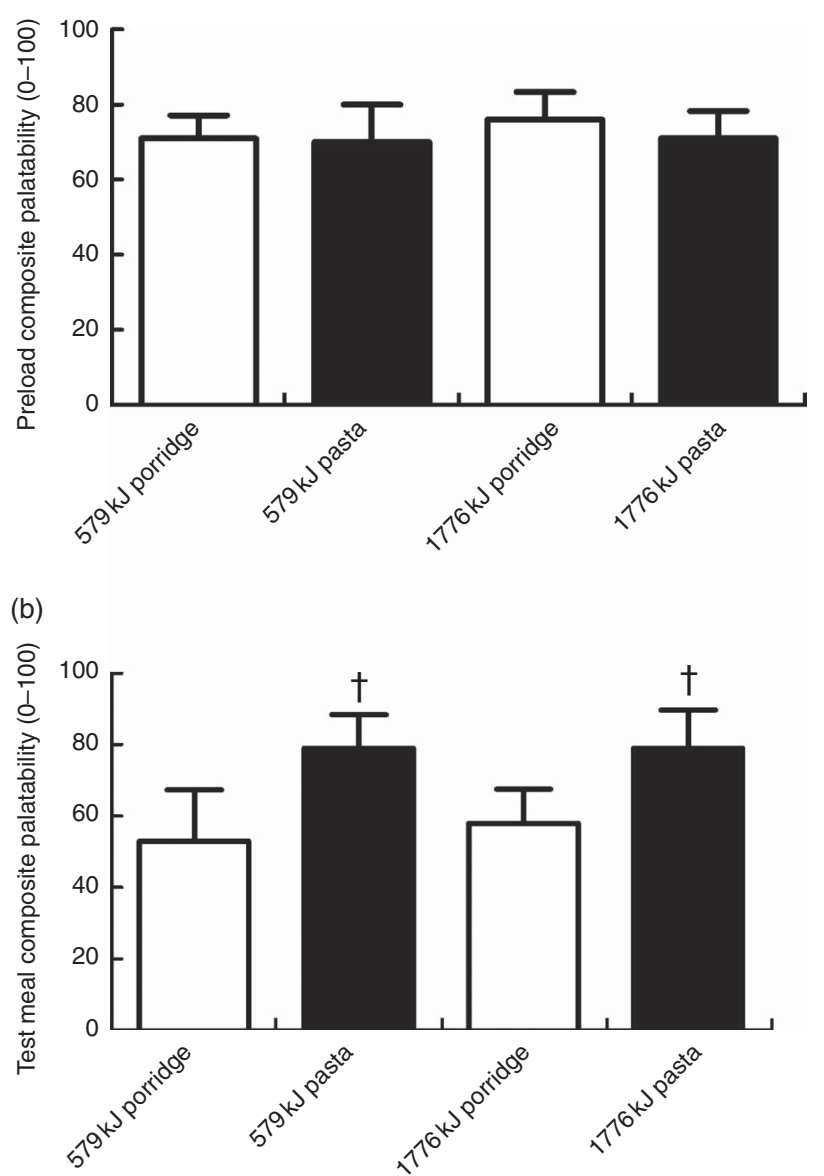

Fig. 3. Composite palatability scores for the preloads (a) and test meals (b) for Expt 1. † Significantly different between test meals. Values are means $(n 10)$ and $95 \% \mathrm{Cl}$ represented by vertical bars.

with the $4188 \mathrm{~kJ}$ preload $(P=0.002)$ and during the pasta meal compared with the porridge meal $(P=0 \cdot 001)$ (Fig. 4(a)). However, there was no significant difference in energy compensation between test meals $(P=0 \cdot 172)$ (Fig. 4(b)).

Appetite and palatability ratings. Two-way ANOVA demonstrated similar results for each appetite perception with higher hunger $(P=0.066)$, higher PFC $(P=0.035)$, lower fullness $(P=0.062)$ and lower satisfaction $(P=0.077)$ after consumption of the $828 \mathrm{~kJ}$ preload compared with the $4188 \mathrm{~kJ}$ preload. There were no significant differences for any of the appetite perceptions between the pasta and the porridge trials (hunger: $P=0.531$; satisfaction: $P=0.813$; fullness: $P=0.654$; PFC: $P=0 \cdot 327)$. Subsequently, composite appetite scores are presented for clarity.

Composite appetite scores did not differ between trials at baseline $(P=0.642)$. Two-way ANOVA revealed higher composite appetite AUC after consumption of the $828 \mathrm{~kJ}$ preload compared with the $4188 \mathrm{~kJ}$ preload $(P=0.051)$. Appetite AUC responses to the preloads did not differ between pasta and porridge trials ( $P=0.642$; LE porridge 69 (sD 9),
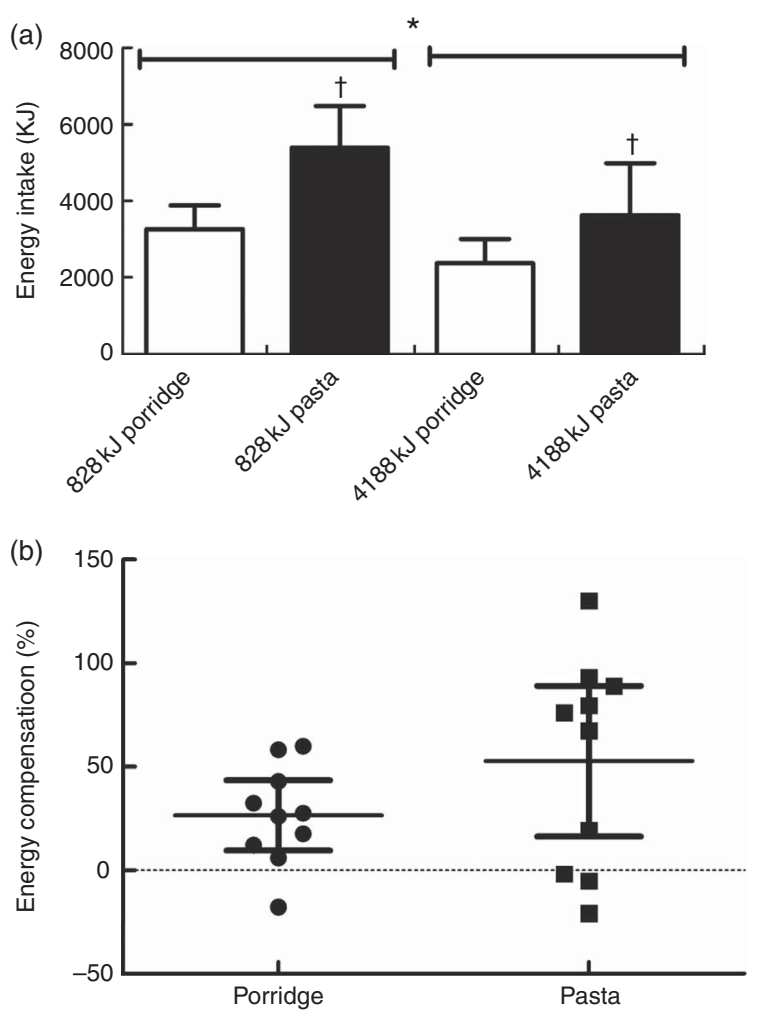

Fig. 4. Energy intake (a) and energy compensation (b) for Expt 2. * Significantly different between preloads, $†$ significantly different between test meals. Values are means $(n 10)$ and $95 \% \mathrm{Cl}$.

LE pasta 66 (SD 13), HE porridge 57 (SD 18), HE pasta 58 (sD 20)) (Fig. 5).

Two-factor ANOVA demonstrated higher composite palatability scores for the $4188 \mathrm{~kJ}$ preload compared with the $828 \mathrm{~kJ}$ preload $(P=0.001)$. The palatability response to preloads was not different during the pasta and porridge trials $(P=0.877)$. Composite palatability scores for the test meals were significantly higher for the pasta meal compared with the porridge meal $(P=0.002)$. The palatability response to the test meals was not different during the low- and high-energy preload trials $(P=0 \cdot 888)$ (Fig. 6).

The preload beverage was correctly identified by the participant in twenty-six of the forty trials and by the researcher in fifteen of the forty trials.

Correlations. Composite appetite AUC values were more strongly correlated with energy intake during the pasta trials than the porridge trials (LE porridge: $r 0 \cdot 165, P=0 \cdot 649$; LE pasta: $r 0.567, P=0.087$; HE porridge: $r 0.565, P=0.089$; HE pasta: $r$ 0.909, $P<0.0005)$. Energy compensation at the ad libitum meal was significantly correlated with the change in AUC and 60-min composite appetite scores between the 828 and the $4188 \mathrm{~kJ}$ preloads for the pasta meal (AUC: $r-0 \cdot 758, P=0 \cdot 011$; $60 \mathrm{~min}$ : $r-0.673, P=0.033$ ), demonstrating greater energy compensation in response to larger reductions in appetite. However, these correlations did not reach statistical significance 

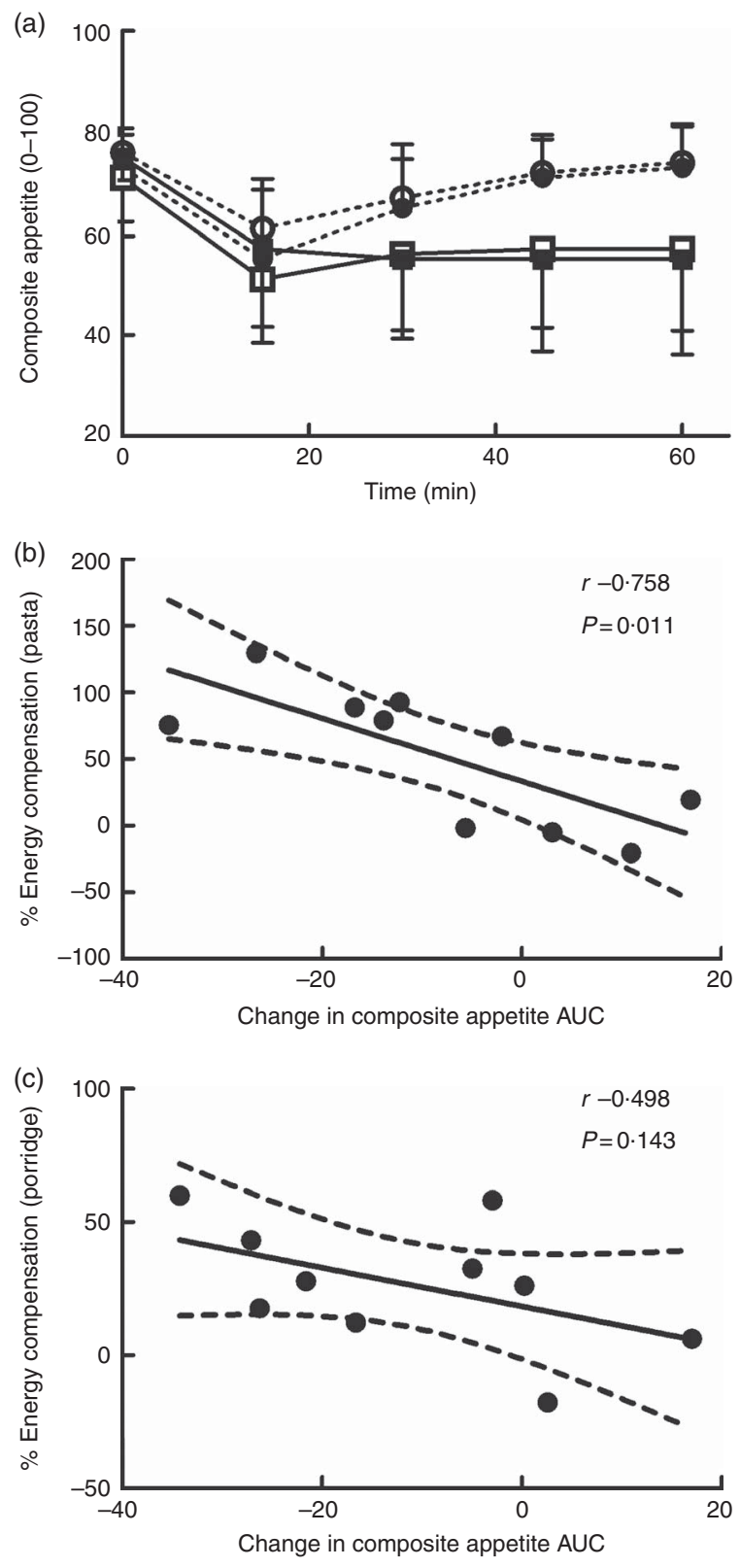

Fig. 5. Composite appetite scores (a) in the $828 \mathrm{~kJ}$ porridge (O), $828 \mathrm{~kJ}$ pasta (@), $4188 \mathrm{~kJ}$ porridge $(\square)$ and $4188 \mathrm{~kJ}$ pasta $(\square)$ trials for Expt 2. - - - -, The low energy preload trials. Values are means $(n 10)$ and $95 \% \mathrm{Cl}$. Linear correlation with $95 \% \mathrm{Cl}$ between the change in composite appetite AUC after the $4188 \mathrm{v} .828 \mathrm{~kJ}$ preload and energy compensation for the pasta meal (b) and porridge meal (c).

for the porridge meal (AUC: $r-0.498, P=0.143$; $60 \mathrm{~min}$ : $r-0.499 ; P=0 \cdot 142$ ) (Fig. 5).

\section{Discussion}

The use of ad libitum meals to quantify energy intake is a prominent methodology within appetite and energy balance research. This investigation represents the first comparison of the sensitivity of two commonly used single-course, ad libitum meals in response to appetite manipulation. These findings
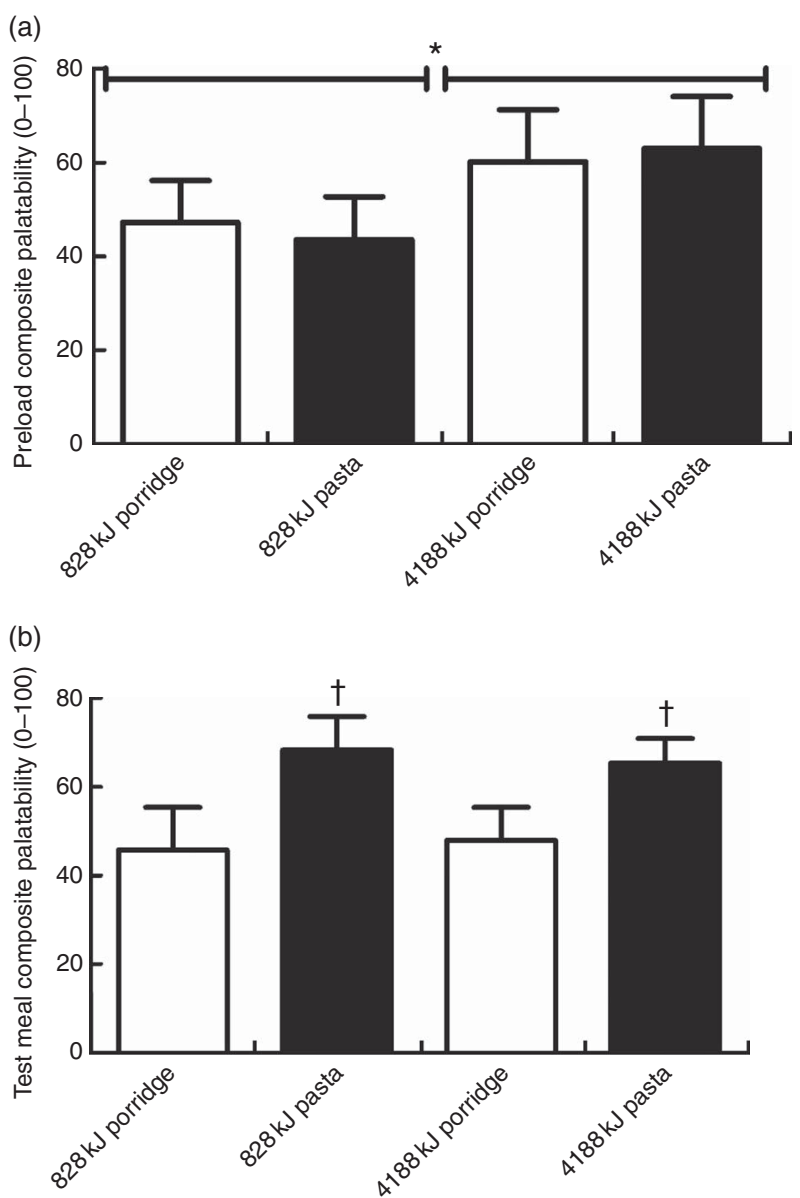

Fig. 6. Composite palatability scores for the preloads (a) and test meals (b) for Expt 2. * Significantly different between preloads, $\dagger$ significantly different between test meals. Values are means $(n 10)$ and $95 \% \mathrm{Cl}$ represented by vertical bars.

demonstrate that the provision of a moderately palatable porridge-based meal reduces overconsumption in comparison with a more highly palatable, pasta-based meal. However, energy compensation at the pasta meal was more strongly correlated with preceding appetite ratings, demonstrating greater sensitivity to appetite manipulation.

The incorporation of two experiments within this report enabled the sensitivity of the test meals to be investigated in response to a moderate and large manipulation of preload energy content. Surprisingly, the $1197 \mathrm{~kJ}$ difference in energy content between preloads in Expt 1 did not produce any discernible changes in appetite or energy intake. This finding contrasts with previous studies that have reported reductions in appetite and an energy intake compensation of $30-57 \%$ in response to preload energy manipulations of approximately $1500 \mathrm{~kJ}^{(19,30)}$. The participants recruited for the present experiment were all young, healthy, recreationally active men, and an intermeal interval of $60 \mathrm{~min}$ was used based on evidence that this population and experimental design will maximise the compensatory response to preload manipulation ${ }^{(19,26,31,32)}$. Subsequently, it is not clear why the preload manipulation failed to alter appetite responses, but this may be related to the 
composition of the preload beverages. In this regard, although similar preload beverages have been found to influence appetite and energy intake through the manipulation of maltodextrin content ${ }^{(19,33)}$, the increases in preload energy during the present study were primarily achieved via the addition of maltodextrin and single cream. Such sugar-fat combinations are frequently used in laboratory models to promote hyperphagia ${ }^{(34)}$, and any appetite-stimulating properties of the higher energy preload may have compensated for the appetite-suppressing effects of the moderately increased energy content. This finding supports long-standing concerns regarding the weak satiating effects of high sugar- and fat dairy-based beverages and their likely contribution to a positive energy balance ${ }^{(35)}$.

The increased manipulation of preload energy content in Expt 2 successfully generated divergent appetite and energy intake responses between the high- and low-energy preloads. Compensatory reductions in energy intake during both ad libitum meals after consumption of the high-energy preload in Expt 2 and the absence of change in energy intake during both meals in Expt 1 support the use of these meals to reflect preceding appetite ratings. However, the findings of the present study reveal important strengths and limitations for the use of these meals in future appetite studies.

In accordance with previous research, the pasta-based, ad libitum meal induced significant overconsumption in both experiments $^{(1,14-20)}$, which conflicts with current recommendations for ad libitum meals to reflect habitual energy intakes ${ }^{(7)}$. In this regard, energy intakes during the pasta meals were more than $50 \%$ higher than the respective porridge meals and occurred despite the meals being matched for energy density. This difference appears to be due to the highly palatable nature of the pasta-based meal and is supported by previous studies demonstrating that highly palatable foods can stimulate appetite during ad libitum feeding, thereby overriding signals of satiation and increasing energy intakes ${ }^{(22,36)}$. The moderately palatable porridge meal produced energy intakes that were more representative of expected habitual intakes, which demonstrated the importance of considering and reporting the palatability ratings of ad libitum meals within research studies. In addition, such large differences in intakes occurred despite participants having higher habitual intakes of pasta-based meals, which would be expected to improve the environmental contingencies associated with this food and reduce intakes to more ecological levels. This further emphasises the importance of palatability as a determinant of energy intake during ad libitum feeding.

Although large inter-individual variation in short-term energy compensation has been previously documented ${ }^{(19,30,37)}$, the findings of the present study suggest that this may be accentuated by the provision of a highly palatable ad libitum meal in response to appetite manipulation. In this regard, higher energy intakes during the pasta meal were associated with markedly greater heterogeneity in the compensatory response to preload manipulation in Expt 2. It seems likely that the higher energy intakes of the pasta meal provided opportunity for greater compensatory responses (i.e. larger changes in energy intake) to the observed decrease in appetite perceptions. Alternatively, the modest energy intakes observed during the porridge meal after consumption of the low-energy preload appear to have limited the potential range available for reductions in energy intake in response to the large manipulation of preload energy content in Expt 2 and produced a more homogenous response. In this regard, although participant blinding was unsuccessful, the participants were unaware of the energy content of the preloads, which maintained the impact of environmental contingencies on food intake and encouraged consumption during both meals ${ }^{(38)}$. Such unsuccessful blinding is an expected consequence of the experimental manipulation as the preload beverages were designed to produce contrasting appetite responses. Although subtle differences in preload appearance may have contributed to the observed appetite responses $^{(39)}$, the successful blinding of experimenters presenting the beverages suggests that post-ingestive consequences from preload consumption may have dominated.

Despite overconsumption and high levels of heterogeneity in compensatory energy intake responses, energy compensation during the pasta-based meal was strongly correlated with appetite changes in response to the high-energy $v$. low-energy preload (i.e. larger reductions in appetite were associated with greater energy compensation). Furthermore, this was superior to the correlations observed between changes in appetite and the more ecologically valid energy intakes achieved during the porridge meal. These findings suggest that the increased range available for compensatory feeding responses as a result of the overconsumption of a highly palatable meal may enhance the sensitivity to reflect preceding appetite ratings and improve alignment between these variables. Subsequently, despite current recommendations for ad libitum meals to reflect habitual energy intakes ${ }^{(7)}$, the present study provides evidence that this may limit the sensitivity of the meal to reflect preceding changes in appetite. However, it must be acknowledged that mean energy compensation was not different between the test meals, which suggests that both meals are sufficiently sensitive to detect compensatory responses to appetite manipulation.

In conclusion, the experiments conducted within this investigation have demonstrated compensatory changes in energy intake in response to appetite manipulation when assessed using either a pasta-based or a porridge-based, ad libitum meal. The provision of a highly palatable, pastabased meal induced significant overconsumption, but changes in energy intake were strongly correlated with preceding appetite ratings. Alternatively, the ecologically valid energy intakes achieved with the provision of a moderately palatable, porridge-based meal were less representative of changes in appetite perceptions. These findings support continuation in the use of a commonly used, pasta-based, ad libitum meal when the priority is to reflect preceding appetite ratings, and suggest that the large energy intakes observed during such feedings are unlikely to reduce the sensitivity of the measure to reflect preceding changes in appetite. Alternatively, it seems that meals producing moderate energy intakes during ad libitum feeding may limit the range of potential compensatory responses but could be suitable when energy intakes reflective of habitual diet are preferable. Subsequently, future ad libitum meal designs may require a compromise between sensitivity and ecological validity. 


\section{Acknowledgements}

The authors thank all the volunteers for their participation in this study.

Data collection at Leeds Beckett University was supported internally by the Carnegie Research Fund. This research received no specific grant from any external funding agency or from commercial or not-for-profit sectors.

All authors contributed to the study design, data collection, data analysis and writing of the manuscript.

The authors declare that there are no conflicts of interest.

\section{References}

1. Chowdhury EA, Richardson JD, Tsintzas K, et al. (2016) Effect of extended morning fasting upon ad libitum lunch intake and associated metabolic and hormonal responses in obese adults. Int J Obes 40, 305-311.

2. Sun FH, Li C, Zhang YJ, et al. (2016) Effect of glycemic index of breakfast on energy intake at subsequent meal among healthy people: a meta-analysis. Nutrients $\mathbf{8}$, E37.

3. Batterham RL, Cowley MA, Small CJ, et al. (2002) Gut hormone PYY(3-36) physiologically inhibits food intake. Nature 418, 650-654.

4. Lippl F, Erdmann J, Steiger A, et al. (2012) Low-dose ghrelin infusion - evidence against a hormonal role in food intake. Regul Pept 174, 26-31.

5. Deighton K \& Stensel DJ (2014) Creating an acute energy deficit without stimulating compensatory increases in appetite: is there an optimal exercise protocol? Proc Nutr Soc 73, 352-358.

6. Schubert MM, Desbrow B, Sabapathy S, et al. (2013) Acute exercise and subsequent energy intake: a meta-analysis. Appetite 63, 92-104.

7. Blundell J, de Graaf C, Hulshof T, et al. (2010) Appetite control: methodological aspects of the evaluation of foods. Obes Rev 11, 251-270.

8. Dhurandhar NV, Schoeller D, Brown AW, et al. (2015) Energy balance measurement: when something is not better than nothing. Int J Obes 39, 1109-1113.

9. Arvaniti K, Richard D \& Tremblay A (2000) Reproducibility of energy and macronutrient intake and related substrate oxidation rates in a buffet-type meal. $B r J$ Nutr $\mathbf{8 3}$, 489-495.

10. Gregersen NT, Flint A, Bitz C, et al. (2008) Reproducibility and power of ad libitum energy intake assessed by repeated single meals. Am J Clin Nutr 87, 1277-1281.

11. Horner KM, Byrne NM \& King NA (2014) Reproducibility of subjective appetite ratings and ad libitum test meal energy intake in overweight and obese males. Appetite 81, $116-122$.

12. Lara J, Taylor MA \& Macdonald IA (2010) Is ad libitum energy intake in overweight subjects reproducible in laboratory studies using the preload paradigm? Eur J Clin Nutr 64 , 1028-1031.

13. Tucker AJ, Heap S, Ingram J, et al. (2016) Postprandial appetite ratings are reproducible and moderately related to total day energy intakes, but not ad libitum lunch energy intakes, in healthy young women. Appetite 99, 97-104.

14. Wiessing KR, Xin L, McGill AT, et al. (2012) Sensitivity of ad libitum meals to detect changes in hunger: restricted-item or multi-item testmeals in the design of preload appetite studies. Appetite 58, 1076-1082.
15. Gonzalez JT, Veasey RC, Rumbold PL, et al. (2013) Breakfast and exercise contingently affect postprandial metabolism and energy balance in physically active males. Br J Nutr $\mathbf{1 1 0}$, $721-732$.

16. Clayton DJ, Stensel DJ, Watson P, et al. (2014) The effect of post-exercise drink macronutrient content on appetite and energy intake. Appetite 82, 173-179.

17. Deighton K, Karra E, Batterham RL, et al. (2013) Appetite, energy intake, and PYY3-36 responses to energy-matched continuous exercise and submaximal high-intensity exercise. Appl Physiol Nutr Metab 38, 947-952.

18. Deighton K, Batterham RL \& Stensel DJ (2014) Appetite and gut peptide responses to exercise and calorie restriction: the effect of modest energy deficits. Appetite 81, 52-59.

19. Appleton KM, Martins C \& Morgan LM (2011) Age and experience predict accurate short-term energy compensation in adults. Appetite 56, 602-606.

20. Chowdhury EA, Richardson JD, Tsintzas K, et al. (2015) Carbohydrate-rich breakfast attenuates glycaemic, insulinaemic and ghrelin response to ad libitum lunch relative to morning fasting in lean adults. Br J Nutr 114, 98-107.

21. Roe LS, Kling SM \& Rolls BJ (2016) What is eaten when all of the foods at a meal are served in large portions? Appetite 99, 1-9.

22. Yeomans MR, Lee MD, Gray RW, et al. (2001) Effects of testmeal palatability on compensatory eating following disguised fat and carbohydrate preloads. Int J Obes Relat Metab Disord 25, 1215-1224.

23. Corney RA, Sunderland C \& James LJ (2016) Immediate premeal water ingestion decreases voluntary food intake in lean young males. Eur J Nutr 55, 815-819.

24. Corney RA, Horina A, Sunderland C, et al. (2015) Effect of hydration status and fluid availability on ad-libitum energy intake of a semi-solid breakfast. Appetite 91, 399-404.

25. de Lauzon B, Romon M, Deschamps V, et al. (2004) The threefactor eating questionnaire-R18 is able to distinguish among different eating patterns in a general population. J Nutr 134, 2372-2380.

26. Almiron-Roig E, Palla L, Guest K, et al. (2013) Factors that determine energy compensation: a systematic review of preload studies. Nutr Rev 71, 458-473.

27. Flint A, Raben A, Blundell JE, et al. (2000) Reproducibility, power and validity of visual analogue scales in assessment of appetite sensations in single test meal studies. Int J Obes Relat Metab Disord 24, 38-48.

28. Stubbs RJ, Hughes DA, Johnstone AM, et al. (2000) The use of visual analogue scales to assess motivation to eat in human subjects: a review of their reliability and validity with an evaluation of new hand-held computerized systems for temporal tracking of appetite ratings. Br J Nutr 84, 405-415.

29. Faul F, Erdfelder E, Lang AG, et al. (2007) G*Power 3: a flexible statistical power analysis program for the social, behavioral, and biomedical sciences. Behav Res Methods 39, 175-191.

30. Soenen S \& Westerterp-Plantenga MS (2007) No differences in satiety or energy intake after high-fructose corn syrup, sucrose, or milk preloads. Am J Clin Nutr 86, 1586-1594.

31. Davy BM, Van Walleghen EL \& Orr JS (2007) Sex differences in acute energy intake regulation. Appetite 49, 141-147.

32. Martins C, Kulseng B, Rehfeld JF, et al. (2013) Effect of chronic exercise on appetite control in overweight and obese individuals. Med Sci Sports Exerc 45, 805-812.

33. Long SJ, Hart K \& Morgan LM (2002) The ability of habitual exercise to influence appetite and food intake in response to high- and low-energy preloads in man. Br J Nutr 87, $517-523$. 
34. Avena NM, Rada P \& Hoebel BG (2009) Sugar and fat bingeing have notable differences in addictive-like behavior. J Nutr 139, 623-628.

35. de Graaf C (2006) Effects of snacks on energy intake: an evolutionary perspective. Appetite 47, 18-23.

36. Robinson TM, Gray RW, Yeomans MR, et al. (2005) Test-meal palatability alters the effects of intragastric fat but not carbohydrate preloads on intake and rated appetite in healthy volunteers. Physiol Behav 84, 193-203.
37. Anderson GH, Catherine NL, Woodend DM, et al. (2002) Inverse association between the effect of carbohydrates on blood glucose and subsequent short-term food intake in young men. Am J Clin Nutr 76, 1023-1030.

38. Blundell JE \& King NA (1999) Physical activity and regulation of food intake: current evidence. Med Sci Sports Exerc 31, S573-S583.

39. Chambers L, McCrickerd K \& Yeomans MR (2015) Optimising foods for satiety. Trends Food Sci Tech 41, 149-160. 